\title{
Comparison of Chromium and Iron Distribution in Serum and Urine among Healthy People and Prediabetes and Diabetes Patients
}

\author{
Qi Zhou, ${ }^{1}$ Wenjia Guo, ${ }^{2}$ Yanan Jia, ${ }^{2}$ and Jiancheng Xu $\mathbb{I D}^{2}$ \\ ${ }^{1}$ Department of Pediatrics, First Hospital of Jilin University, Changchun 130021, China \\ ${ }^{2}$ Department of Laboratory Medicine, First Hospital of Jilin University, Changchun 130021, China \\ Correspondence should be addressed to Jiancheng Xu; jianchengxu@yeah.net
}

Received 19 December 2018; Accepted 10 February 2019; Published 24 February 2019

Guest Editor: Filipa S. Reis

Copyright (C) 2019 Qi Zhou et al. This is an open access article distributed under the Creative Commons Attribution License, which permits unrestricted use, distribution, and reproduction in any medium, provided the original work is properly cited.

\begin{abstract}
The effect of chromium $(\mathrm{Cr})$ and iron $(\mathrm{Fe})$ on prevalence of diabetes has received great attention. This study investigated serum and urinary $\mathrm{Cr}$ and Fe levels among patients with impaired fasting glucose (IFG), impaired glucose tolerance (IGT), type 1 diabetes (T1D), and type 2 diabetes (T2D) in the Northeast Chinese population. From January 2010 to October 2011, patients with IFG $(n=12)$, IGT $(n=15)$, T1D $(n=25)$, T2D $(n=137)$ and healthy controls $(n=50)$ were enrolled in the First Hospital of Jilin University. Trace elements were detected using an inductively coupled plasma spectrometer. Serum Cr levels decreased in T2D without complications, diabetic retinopathy (DR), diabetic peripheral neuropathy (DPN), and diabetic nephropathy $(\mathrm{DN})(P<0.05)$. The urinary $\mathrm{Cr}$ level in T1D was the highest of all, which significantly exceeded those of the T2D groups with and without complications. No significant differences of serum Fe levels were found among all groups. The urinary Fe level of T1D was significantly increased $(P<0.05)$. The correlation between serum $\mathrm{Cr}$ and serum Fe in T2D was obviously positive $(P<0.05)$. One month of simvastatin therapy exerted no effects on serum or urinary $\mathrm{Cr}$ and Fe levels. These results suggest the potential role of $\mathrm{Cr}$ and $\mathrm{Fe}$ in diabetes should receive attention.
\end{abstract}

\section{Introduction}

Incidence of diabetes mellitus (DM) has been remaining high worldwide. The chronic disease has caused 1.6 million people to die in 2015 [1] and WHO predicts the number of patients would be over 592 million people in 2035 [2]. As the 4thfatal illness of the noncommunicable diseases [1], it worsens patients' health and quality of life severely. Previous studies have shown increasing prevalence of diabetes in China, which is the world's largest diabetes epidemic now.

Among the Chinese adult population in 2013, the estimated standardized prevalence of total diagnosed and undiagnosed diabetes is $10.9 \%$; that of diagnosed diabetes, $4.0 \%$; and that of prediabetes, $35.7 \%$ [3]. Diet plus physical activity may reduce the incidence of type 2 diabetes (T2D) in people with impaired glucose tolerance (IGT).

Basic and clinical studies reveal that transportation, distribution, excretion, and accumulation of various kinds of trace elements under diabetic condition changed differently
[4-6] and influence development of diabetes and complication progression dissimilarly [7]. Considering the nosogenesis mechanism, chromium $(\mathrm{Cr})$ and iron $(\mathrm{Fe})$ promote diabetes through insulin resistance solely $[8,9]$. Some scholars believe Cr benefits the human body [10]. Cr promotes glycolysis in muscle cells and fat cells, acts as an inhibitor of glycogen decomposition in myocytes, and is able to regulate glucose according to a variety of animal models and clinical trials. It is classified as a "hypoglycemic metal element" in the review of Adrian et al. [11], which is involved in carbohydrate, lipid, and protein metabolism primarily by increasing insulin efficiency [12]. Chromium-containing compounds enhance insulin activity, and what is approved is that insulin-responsiveness is improved in diabetic patients after supplementation with chromium-based compounds [13]. Its mechanism may be increasing the concentration of the insulin receptor RNA messenger; complex formation with insulin enhancing its activity; and increasing islet $\beta$ cell sensitivity by reducing concentration of TNF- $\alpha$, resistin, and 
interleukin-6 [14]. Many clinical studies have confirmed the decrease of serum $\mathrm{Cr}$ in patients with T1D and T2D $[15,16]$ with a drastic increase of $\mathrm{Cr}$ in urine [17]. Plasma $\mathrm{Cr}$ is negatively correlated with glucose in patients with T2D [18].

$\mathrm{Fe}$ is an essential element of organisms, a constituent of various proteins and enzymes. There has been no experiment so far proving that $\mathrm{Fe}$ directly increases glucose, while evidence shows it damages the pancreas and induces diabetes [11]. It is currently believed that Fe overload strongly associates with insulin resistance, hyperglycemia, and high risk of T2D [19]. For example, ferritin, the index for Fe storage, is correlated with diabetes [20]. Some researchers believe that $\mathrm{Fe}$ induces diabetes through oxidative stress and direct damage towards pancreatic $\beta$ cells [21]. In addition, the other side is that frequent blood donation ( $\geq 2$ times per year) is considered to be a protective factor for diabetes, which reduces Fe reserve, increases insulin sensitivity, and weakens postprandial hyperinsulinemia [22].

The function of each element is different and their relationships appear to be complex, as calcium, magnesium, titanium, zinc, vanadium, and Fe reduce $\mathrm{Cr}$ absorption [23]. Therefore many investigators now turn to "pack" them to analyze the changes and roles of several more related ones. We have done so as with magnesium and calcium, copper, and zinc $[5,6]$. Because both $\mathrm{Cr}$ and $\mathrm{Fe}$ function as an insulin resistant, we conduct this comparative study on the distribution and correlation of $\mathrm{Cr}$ and Fe among healthy, prediabetic, and diabetic populations and try to explore the interaction of the two. By exploring their levels in the serum and urine of subjects, respectively, we also intend to track metabolic changes and disease-relevant information subsequently.

\section{Materials and Methods}

2.1. Ethical Statements. This retrospective study was approved by the Ethics Committee of the First Hospital of Jilin University. All patients provided signed informed consent. Data were obtained from electronic medical records of the hospital, and the information was anonymous.

2.2. Subjects. To ensure academic integrity and rigor, subject selection of this study is the same as those of our previous researches $[5,6]$, which can be described briefly as 189 definitively diagnosed patients enrolled from January 2010 to October 2011 in the First Hospital of Jilin University and grouped based on medical certification as follows: impaired fasting glucose (IFG, $n=12$ people), impaired glucose tolerance (IGT, $n=15$ ), type 1 diabetes (T1D, $n=25$ ), type 2 diabetes without complications (T2D, $n=29$ ), diabetic nephropathy (DN, $n=24)$, diabetic retinopathy (DR, $n=34$ ), and diabetic peripheral neuropathy (DPN, $n=50)$. The control group (CON, $n=50)$ was enrolled according to the age and sex proportion from physical examination group at the same standard meantime, noting that subjects had not received any element supplement due to official approval of effectiveness. The subjects' basic information as age, sex, and BMI will not be detailed here to avoid redundancy and duplication [5].
2.3. Element Measurement. The elements' measurements were the same as previous studies of our research project $[5,6]$, using ICP-MS. The quality control of all analyzed samples was performed by using standard reference materials from the China Standard Material Center. Limits of detection (LOD) were $1.0 \mu \mathrm{g} / \mathrm{L}$ for $\mathrm{Cr}$ and Fe. The recovery of standard trace elements (accuracy) ranged from $93.3 \%$ to $98.9 \%$.

2.4. Statistics. Statistical description was performed as median (interquartile range); the Kruskal-Wallis test was used for evaluation among multigroups, and the comparison between groups was assessed applying the Mann-Whitney test with correction by the Bonferroni method of $\alpha$; correlation was analyzed with Spearman's method. The $P$ value reported was two-sided and statistically significant at $P$ $<0.05$. All analysis was conducted using SPSS 24.0.

\section{Results and Discussion}

3.1. Trace Elements Levels in Prediabetes and Diabetes. Serum glucose and HbAlc were tested to support the enrollment standard. Table 1 showed that, compared with the control group, serum Cr levels decreased in the T2DCON, DR, DPN, and DN groups $(P<0.05)$; though no statistical significance $(P>0.05)$ was shown, a downward trend appeared in IFG, IGT, and T1D. On the other hand, the urinary Cr level in T1D was the highest of all (Figure 1(a)), which significantly exceeded those of the T2D groups with and without complications. Surprisingly, we barely found significant difference of serum Fe levels among all groups. The urinary Fe level of T1D was significantly increased $(P<0.05)$ and reached the peak (Figure $1(\mathrm{~b})$ ), while that of T2D was lower $(P<0.05)$.

3.2. Correlation Analysis of Trace Elements. As presented in Table 2, the correlation between serum $\mathrm{Cr}$ and serum $\mathrm{Fe}$ in T2D was positive obviously $(P<0.05)$; meanwhile these relations in IFG, IGT, and T1D were not statistically significant $(P>0.05)$.

3.3. Effect of Simvastatin Treatment on Trace Elements in T2D Patients. Statin treatment was often used to lower lipid in the patients with T2D. Therefore the effect of simvastatin therapy for one month on serum or urinary $\mathrm{Cr}$ and Fe levels was detected in the patients with T2D. As presented in Table 3, one month of simvastatin therapy had no effects on serum or urinary $\mathrm{Cr}$ and $\mathrm{Fe}$ levels.

\section{Discussion}

4.1. Comparison of Serum and Urinary Cr. It is currently believed that diabetic patients are under a state of trace elements disorder, such as insulin promoting discrepancy of distribution of multiple ions in the bloodstream [4]. More than $80 \%$ of the $\mathrm{Cr}$ in the human body is excreted in urine [24], and the amount of urinary $\mathrm{Cr}$ in T1D patients is considered to be more than twice that of the control group [17]. Some scientists attributed this to its increased loss plus decreased absorption [25]. Therefore, we believe the 


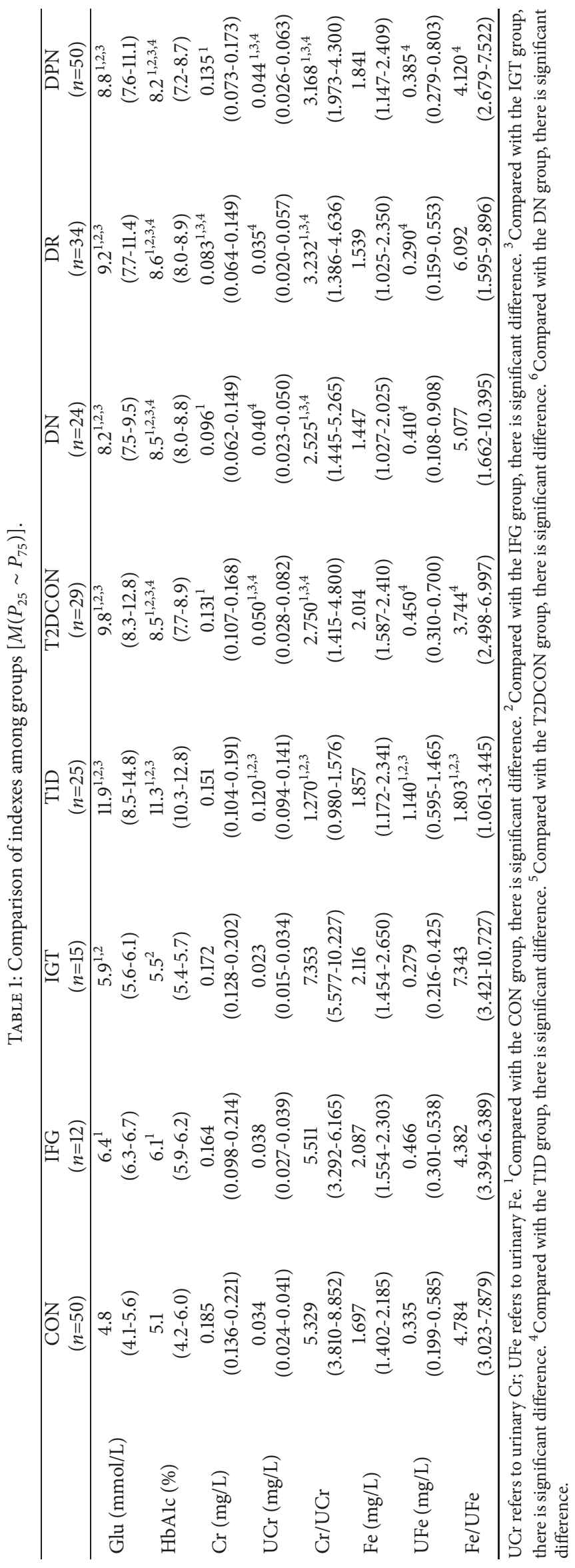




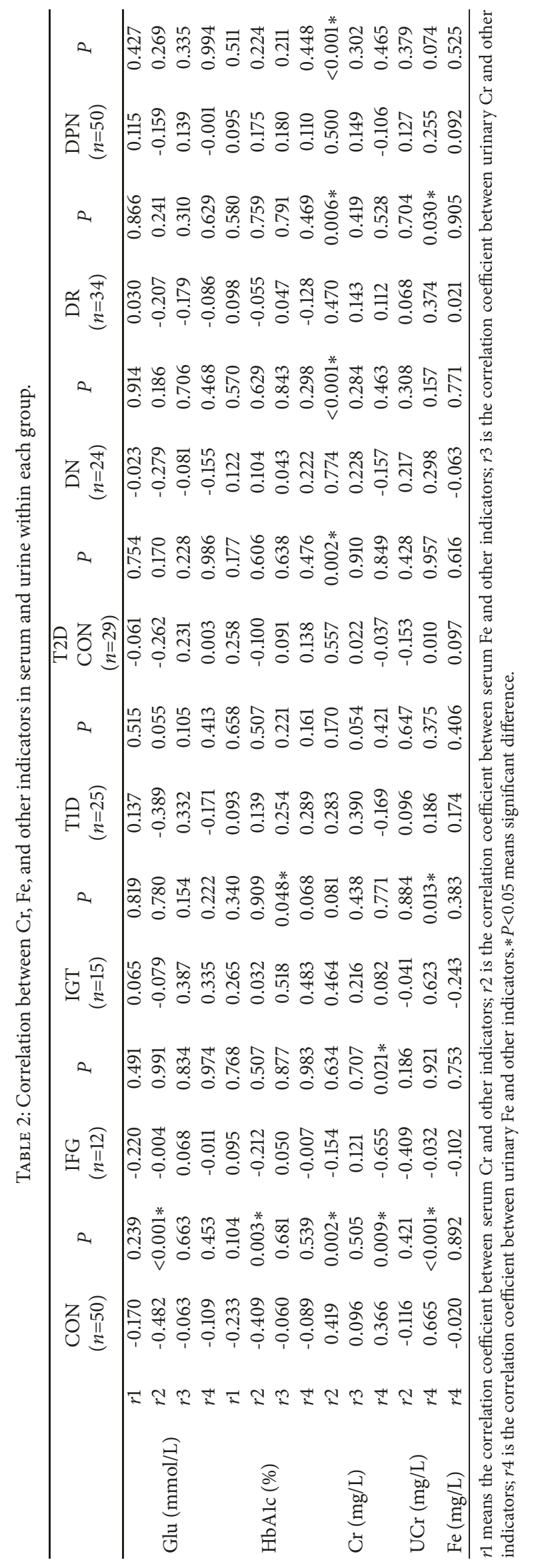


TABLE 3: Serum parameters in T2D patients treated with simvastatin.

\begin{tabular}{lccc}
\hline & & Simvastatin $(n=24)$ & \\
& Pretreatment & Posttreatment & $P$ \\
\hline Cr & $0.165(0.087-0.241)$ & $0.178(0.067-0.234)$ & 0.695 \\
UCr & $0.048(0.020-0.064)$ & $0.034(0.021-0.057)$ & 0.837 \\
Fe & $2.235(1.461-2.742)$ & $2.147(1.685-2.852)$ & 0.680 \\
UFe & $0.370(0.173-0.735)$ & $0.320(0.213-0.770)$ & 0.724 \\
\hline
\end{tabular}

Data presentation and abbreviations' spelt-out forms are the same as the description for Table 1.

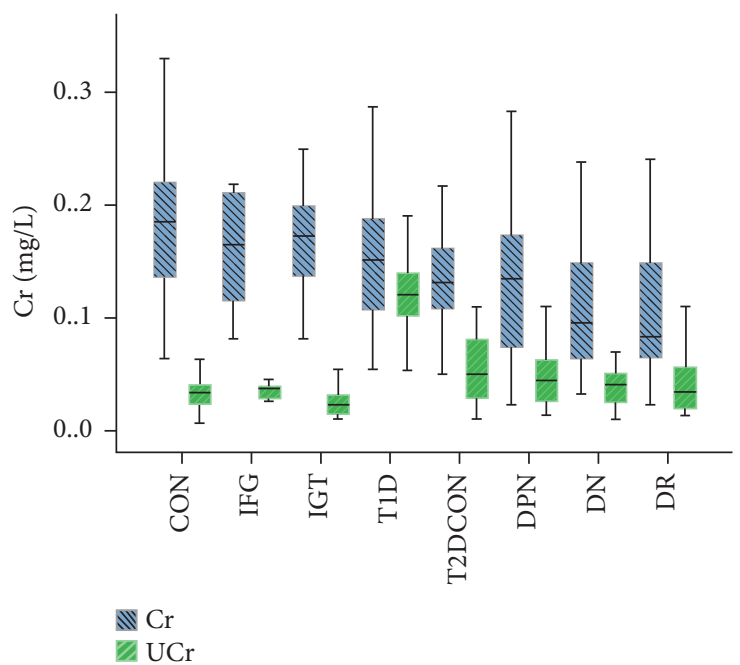

(a)

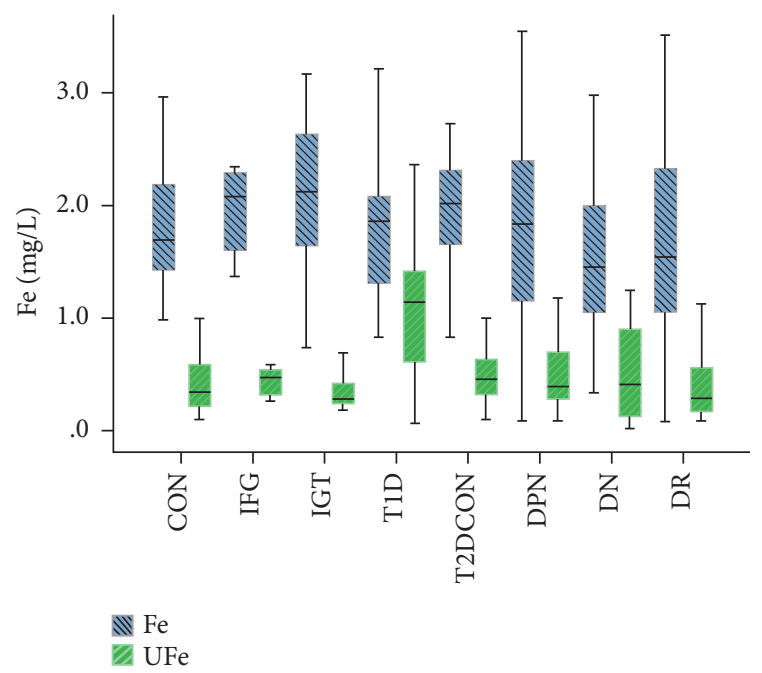

(b)

FIGURE 1: Levels of $\mathrm{Cr}$ and Fe in the healthy control, IFG, IGT, T1D, T2D without complications, DPN, DN, and DR groups. Boxplots display the extreme, the upper and lower quartiles, and the median of the maximum difference in the healthy control, IFG, IGT, T1D, T2D without complications, DPN, DN, and DR groups. The median for each dataset is indicated by the centerline, and the first and third quartiles are presented by the edges of the area, which is known as the interquartile range (IQR). (a) Serum Cr levels decreased in the T2DCON, DR, DPN, and DN group $(P<0.05)$ and urinary Cr level in T1D was the highest; (b) urinary Fe level of T1D was significantly increased $(P<0.05)$.

simultaneous study of both serum and urine trace element levels may provide better overview of the state and degree. We confirmed serum $\mathrm{Cr}$ levels were lower in the T2DCON, DR, $\mathrm{DPN}$, and DN population than that of the control, which is consistent with findings of other investigators [7, 26, 27]; IFG and IGT had lower serum Cr levels than the control even with no statistical significance, which is similar to the conclusion of Chen [27]; the comparison of serum Cr between T1D and the control showed no significant difference and was consistent with Lin's study [28] of T1D children, adults, and healthy people.

As for urinary $\mathrm{Cr}$, the sum that $\mathrm{T} 2 \mathrm{D}$ patients have higher levels of urinary $\mathrm{Cr}$ was the same as the proposition of Kazi et al. [7] who proved no statistically significant difference as well but deemed the tendency existed; it elevated visibly in T1D; considering the severity of illness and thus selection being incomprehensive, prediabetes showed no significant difference comparing with the control group on urinary $\mathrm{Cr}$ level; the $\mathrm{Cr} / \mathrm{UCr}$ in diabetic groups was lower than those of the prediabetic and control group, indicating diabetic patients were all under low $\mathrm{Cr}$ state. It is already proved in a research launching in Indian rural areas that elevated urinary Cr is strongly correlated to diabetes [29]; our experiment adopted a random urine specimen, which might have varied greatly due to physical and chemical factors; thus the statistical difference could not be that obvious. Besides, it is noticeable that the excretion rate of $\mathrm{Cr}$ and $\mathrm{Fe}$ changed mildly in DN patients, which was opposite to our expectation that severe renal function damaged influenced elements in urine.

Combined with serum $\mathrm{Cr}$ information, it was stated in our experiment that T2D patients lied in low $\mathrm{Cr}$ condition, the same as findings of relatively low $\mathrm{Cr}$ content in T2D patients' hair [7] and nails [30]. That explained what Cefalu WT [31] purposed in which low $\mathrm{Cr}$ induced upward fasting glucose and glycosuria. T1D patients exhibited a negative $\mathrm{Cr}$ balance [32], which was in consistency with the results of no significant change of serum $\mathrm{Cr}$ and overt increase of urinary $\mathrm{Cr}$ in our study. It is stated that the T1D metabolic control system required additional $\mathrm{Cr}$ whereas the absorbed element was not utilized and discharged in urine [17].

Besides, $\mathrm{Cr} / \mathrm{UCr}$ results also revealed that diabetic patients were losing Cr. Morris [18] found diabetic patients had a 33\% reduction of plasma $\mathrm{Cr}$ and $100 \%$ increase of urinary Cr. Our experiment could not find stationary correlation between $\mathrm{Cr}$ and lipid indicators which was testified in a lot of 
animal and clinical studies $[4,31,33,34]$, possibly resulting from therapeutic regimens and medications.

As an enhancer for insulin function, Cr participates in carbohydrate, lipid, and protein metabolism by improving insulin efficiency, and its deficiency seriously affects glucose tolerance and lipid homeostasis [12]. The concrete mechanism remains unknown [12], whereas studies confirmed $\mathrm{Cr}$ regulated glucose in a variety of pathways. For example, $\mathrm{Cr}$ is involved in hyperinsulinemia and insulin resistance [35]; Vincent et al. [36] demonstrated that chromodulin was able to activate insulin receptor kinase; Pattar et al. [37] believed $\mathrm{Cr}$ altered membrane fluidity and regulated glucose uptake consequently by changing cholesterol content; it might impact structure of lipid bilayer [38] and the like. Both animal experiments [39] and clinical studies [40-42] have affirmed that $\mathrm{Cr}$ supplementation reduced glucose; therefore experts suggested [43] that that be applied as T2D complementary therapy.

However, healthy people did not benefit from that tentative plan [44]. Therefore, Lewicki S [12] thought this treatment was probably only valid to a population with metabolic disorders, which should be supplemented as appropriate.

4.2. Comparison of Serum and Urinary Fe. Surprisingly, there was no significant difference of serum Fe levels among the groups in our study, which was contrary with others' $[45,46]$. Most current views were that diabetic patients are being Fe overloaded; evidence stood as diabetes associating with elevated ferritin [47]. Yet some data turned out that serum Fe level was not distinctive between diabetic patients and healthy people [15, 47-49]. Duan et al. [47] considered hyperglycemia led to enhancing oxidative stress that reduced free iron in circulation, with elevated hepcidin inhibiting the intestine absorbing $\mathrm{Fe}$ and release of $\mathrm{Fe}$ by the reticuloendothelial system; the correlation between serum $\mathrm{Cr}$ and serum $\mathrm{Fe}$ in IFG and IGT was not significant, but we still found the tendency existed. Therefore it might be ascribed to small sample size or case selection. Urinary Fe in T1D increased significantly and was much higher than that of T2D and the control. Additionally, Fe/UFe in T1D was apparently lower than other groups', indicating more Fe excretion. In the present study, serum Fe of DPN was not very disparate from that of CON and DN; the DPN and DR group encountered the same situation, which might result in no significant discrepancy. In the present study, one month of simvastatin therapy had no effects on serum or urinary Fe levels.

4.3. Correlation of $\mathrm{Cr}$ and $\mathrm{Fe}$. Because of wide variety and complex mechanism of trace elements in the body, their mutual correlation and influence are still inconclusive in this emerging field. We have observed a significant correlation between serum $\mathrm{Cr}$ and serum $\mathrm{Fe}$, in accordance with previous findings from supplement experiments [50, 51], possibly due to the cotransport mechanism of $\mathrm{Cr}$ and $\mathrm{Fe}$ resulting competition for binding sites of transferrin. In vivo and in vitro studies in rats have shown that approximately $80 \%$ of the $\mathrm{Cr}$ in the blood is associated with transferrin [52]. After being absorbed from the intestinal tract, $\mathrm{Cr}$ combines with proteins related to Fe metabolism, forming a complex transported into cells. The efficiency of the compound passing through the membrane depends on insulin concentration [53]. The bioavailability of Fe in rats treated with $\mathrm{Cr}$ intraperitoneally was reduced, and the animal even developed symptoms of anemia [54].

Our experiment concluded that patients with T2D were in low $\mathrm{Cr}$ state. The levels of urinary $\mathrm{Cr}$ and Fe in T1D patients were higher than those of T2D patients. It is suspected that loss and degree of disorder of trace elements in T1D patients are more severe. We assumed that diabetes affected absorption, transportation, and utilization of $\mathrm{Cr}$ and Fe. The limitations of this experiment are that the number of enrolled cases was not enough and the prediabetic group consisted of outpatients; therefore it would be difficult to follow up; there is no more detailed research, such as disease-staging patients; in a way, a $24 \mathrm{~h}$ urine sample may be better than a random urine specimen, which reflects cumulative exposure, exposure approaches, and different forms of elements [29]; metabolism, lifestyle, or drugs may not be excluded from impact of exposure, absorption, or excretion of certain elements.

We have not found any significant difference after simvastatin treatment. However, the data presented that serum $\mathrm{Cr}$ elevated and serum Fe and urinary $\mathrm{Cr}$ and Fe decreased after treatment. The efficacy might be obvious and statistically significant if the duration was set longer.

\section{Conclusions}

Trace elements and diabetes affect each other mutually. Research is now clinically focused on supplement effectiveness $[55,56]$ and control glucose as a result $[47,48]$. For the reason that it has not yet accessibly arrived at an evidencebased aspect, there is no final conclusion concerning safety and availability [56]. What remains a current and difficult point in future research is to accurately extract and analyze the relationship and mechanism of synergy and antagonism through ingenious experimental design and research.

\section{Data Availability}

The data used to support the findings of this study are available from the corresponding author upon request.

\section{Conflicts of Interest}

The authors declare that there are no conflicts of interest regarding the publication of this paper.

\section{Acknowledgments}

We are thankful for Suyan Tian and Zhifang Jia for providing statistical advice. The data cited from the laboratories of the authors were supported in part by grants from the National Science Foundation of China (no. 81501839, to Dr. Qi Zhou), Scientific and Technological "13th Five-Year 
Plan" Project of Jilin Provincial Department of Education (no. JJKH20180214KJ, to Dr. Qi Zhou), Jilin Province Health and Technology Innovation Development Program (no. 2017J071, to Dr. Jiancheng Xu), the Jilin Science and Technology Development Program (no. 20170623092TC-09, to Dr. Jiancheng Xu; no. 20160101091JC, to Dr. Jiancheng Xu; no. 20150414039 GH, to Dr. Jiancheng Xu; no. $20190304110 Y Y$, to Dr. Jiancheng Xu), the First Hospital Translational Funding for Scientific \& Technological Achievements (no. JDYYZH1902002, to Dr. Jiancheng Xu), and Norman Bethune Program of Jilin University (no. 2012223, to Dr. Jiancheng Xu).

\section{References}

[1] World Health Organization, "World health statistics," 2017.

[2] World Health Organization, "Global report on diabetes," WHO, 2016.

[3] L. Wang, P. Gao, M. Zhang et al., "Prevalence and ethnic pattern of diabetes and prediabetes in china in 2013," Journal of the American Medical Association, vol. 317, no. 24, pp. 2515-2523, 2017.

[4] H. Staniek, N. R. Rhodes, K. R. Di Bona et al., "Comparison of tissue metal concentrations in zucker lean, zucker obese, and zucker diabetic fatty rats and the effects of chromium supplementation on tissue metal concentrations," Biological Trace Element Research, vol. 151, no. 3, pp. 373-383, 2013.

[5] J. Xu, Q. Zhou, G. Liu, Y. Tan, and L. Cai, "Analysis of serum and urinal copper and zinc in chinese northeast population with the prediabetes or diabetes with and without complications," Oxidative Medicine and Cellular Longevity, vol. 2013, Article ID 635214, 11 pages, 2013.

[6] Q. Zhou, W. Guo, Y. Jia, and J. Xu, "Effect of 4-phenylbutyric acid and tauroursodeoxycholic acid on magnesium and calcium metabolism in streptozocin-induced type 1 diabetic mice," Biological Trace Element Research, pp. 1-10, 2018.

[7] T. G. Kazi, H. I. Afridi, N. Kazi et al., "Copper, chromium, manganese, iron, nickel, and zinc levels in biological samples of diabetes mellitus patients," Biological Trace Element Research, vol. 122, no. 1, pp. 1-18, 2008.

[8] S. Fargion, P. Dongiovanni, A. Guzzo, S. Colombo, L. Valenti, and A. L. Fracanzani, "Iron and insulin resistance," Alimentary Pharmacology and Therapeutics, Supplement, vol. 22, Suppl 2, pp. 61-63, 2005.

[9] Y. Hua, S. Clark, J. Ren, and N. Sreejayan, "Molecular mechanisms of chromium in alleviating insulin resistance," The Journal of Nutritional Biochemistry, vol. 23, no. 4, pp. 313-319, 2012.

[10] S. Weksler-Zangen, T. Mizrahi, I. Raz, and N. Mirsky, "Glucose tolerance factor extracted from yeast: oral insulin-mimetic and insulin-potentiating agent: in vivo and in vitro studies," British Journal of Nutrition, vol. 108, no. 05, pp. 875-882, 2012.

[11] A. González-Villalva, L. Colín-Barenque, P. Bizarro-Nevares et al., "Pollution by metals: Is there a relationship in glycemic control?” Environmental Toxicology and Pharmacology, vol. 46, pp. 337-343, 2016.

[12] S. Lewicki, R. Zdanowski, M. Krzyzowska et al., "The role of chromium III in the organism and its possible use in diabetes and obesity treatment," Annals of Agricultural and Environmental Medicine, vol. 21, no. 2, pp. 331-335, 2014.
[13] M. Lai, "Antioxidant Effects and Insulin Resistance Improvement of Chromium Combined with Vitamin C and E Supplementation for Type 2 Diabetes Mellitus," Journal of Clinical Biochemistry and Nutrition, vol. 43, no. 3, pp. 191-198, 2008.

[14] S. K. Jain and K. Kannan, "Chromium chloride inhibits oxidative stress and TNF- $\alpha$ secretion caused by exposure to high glucose in cultured U937 monocytes," Biochemical and Biophysical Research Communications, vol. 289, no. 3, pp. 687691, 2001.

[15] G. Forte, B. Bocca, A. Peruzzu et al., "Blood metals concentration in type 1 and type 2 diabetics," Biological Trace Element Research, vol. 156, no. 1-3, pp. 79-90, 2013.

[16] C. R. Flores, M. P. Puga, K. Wrobel, M. E. G. Sevilla, and $\mathrm{K}$. Wrobel, "Trace elements status in diabetes mellitus type 2: possible role of the interaction between molybdenum and copper in the progress of typical complications," Diabetes Research and Clinical Practice, vol. 91, no. 3, pp. 333-341, 2011.

[17] A. C. Nsonwu, C. A. O. Usoro, M. H. Etukudo, and I. N. Usoro, "Glycemic control and serum and urine levels of zinc and magnesium in diabetics in Calabar, Nigeria," Pakistan Journal of Nutrition, vol. 5, no. 1, pp. 75-78, 2006.

[18] B. Morris, S. MacNeil, C. Hardisty, S. Heller, C. Burgin, and T. Gray, "Chromium homeostasis in patients with type II (NIDDM) diabetes," Journal of Trace Elements in Medicine and Biology, vol. 13, no. 1-2, pp. 57-61, 1999.

[19] J. M. Fernández-Real, A. López-Bermejo, and W. Ricart, “Crosstalk between iron metabolism and diabetes," Diabetes, vol. 51, no. 8, pp. 2348-2354, 2002.

[20] Y. Wang, W. Koh, J. Yuan, and A. Pan, "Plasma ferritin, C-reactive protein, and risk of incident type 2 diabetes in Singapore Chinese men and women," Diabetes Research and Clinical Practice, vol. 128, pp. 109-118, 2017.

[21] M. Hatunic, F. M. Finucane, A. M. Brennan, S. Norris, G. Pacini, and J. J. Nolan, "Effect of iron overload on glucose metabolism in patients with hereditary hemochromatosis," Metabolism, vol. 59, no. 3, pp. 380-384, 2010.

[22] J. M. Fernández-Real, A. López-Bermejo, and W. Ricart, "Iron stores, blood donation, and insulin sensitivity and secretion," Clinical Chemistry, vol. 51, no. 7, pp. 1201-1205, 2005.

[23] N. S. Chen, A. Tsai, and I. A. Dyer, "Effect of chelating agents on chromium absorption in rats," Journal of Nutrition, vol. 103, no. 8, pp. 1182-1186, 1973.

[24] V. Ducros, "Chromium metabolism - A literature review," Biological Trace Element Research, vol. 32, no. 1-3, pp. 65-77, 1992.

[25] J. J. Cunningham, "Micronutrients as nutriceutical interventions in diabetes mellitus," Journal of the American College of Nutrition, vol. 17, no. 1, pp. 7-10, 1998.

[26] G. Sridhar, V. Sujatha, V. Anita, and P. Sundararaman, "Serum chromium levels in gestational diabetes mellitus," Indian Journal of Endocrinology and Metabolism, vol. 16, no. 7, pp. S70-S73, 2012.

[27] S. Chen, X. Jin, Z. Shan et al., "Inverse association of plasma chromium levels with newly diagnosed type 2 diabetes: a casecontrol study," Nutrients, vol. 9, no. 3, 2017.

[28] C. Lin, G. Tsweng, C. Lee, B. Chen, and Y. Huang, "Magnesium, zinc, and chromium levels in children, adolescents, and young adults with type 1 diabetes," Clinical Nutrition, vol. 35, no. 4, pp. $880-884,2016$.

[29] G. Velmurugan, K. Swaminathan, G. Veerasekar et al., "Metals in urine in relation to the prevalence of pre-diabetes, diabetes 
and atherosclerosis in rural India," Occupational and Environmental Medicine, vol. 75, no. 9, pp. 661-667, 2018.

[30] M. Khamaisi, I. D. Wexler, J. Skrha et al., "Cardiovascular disease in type 2 diabetics: epidemiology,risk factors and therapeutic modalities," The Israel Medical Association Journal (IMAJ), vol. 5, pp. 801-806, 2003.

[31] W. T. Cefalu, Z. Q. Wang, X. H. Zhang, L. C. Baldor, and J. C. Russell, "Oral Chromium Picolinate Improves Carbohydrate and Lipid Metabolism and Enhances Skeletal Muscle Glut-4 Translocation in Obese, Hyperinsulinemic (JCR-LA Corpulent) Rats," Journal of Nutrition, vol. 132, no. 6, pp. 1107-1114, 2002.

[32] B. S. Karagun, F. Temiz, G. Ozer et al., "Chromium levels in healthy and newly diagnosed type 1 diabetic children," Pediatrics International, vol. 54, no. 6, pp. 780-785, 2012.

[33] R. Bennett, B. Adams, A. French, Y. Neggers, and J. B. Vincent, "High-dose chromium(iii) supplementation has no effects on body mass and composition while altering plasma hormone and triglycerides concentrations," Biological Trace Element Research, vol. 113, no. 1, pp. 53-66, 2006.

[34] M. H. Lai, Y. Y. Chen, and H. H. Cheng, "Chromium yeast supplementation improves fasting plasma glucose and ldlcholesterol in streptozotocin-induced diabetic rats," International Journal for Vitamin and Nutrition Research, vol. 76, no. 6, pp. 391-397, 2006.

[35] M. P. Longnecker and J. L. Daniels, "Environmental contaminants as etiologic factors for diabetes.," Environmental Health Perspectives, vol. 109, no. suppl 6, pp. 871-876, 2001.

[36] J. B. Vincent, "Mechanisms of chromium action: lowmolecular-weight chromium-binding substance," Journal of the American College of Nutrition, vol. 18, no. 1, pp. 6-12, 1999.

[37] G. R. Pattar, L. Tackett, P. Liu, and J. S. Elmendorf, "Chromium picolinate positively influences the glucose transporter system via affecting cholesterol homeostasis in adipocytes cultured under hyperglycemic diabetic conditions," Mutation Research Genetic Toxicology and Environmental Mutagenesis, vol. 610, no. 1-2, pp. 93-100, 2006.

[38] N. S. Raja, K. Sankaranarayanan, A. Dhathathreyan, and B. U. Nair, "Interaction of chromium(III) complexes with model lipid bilayers: Implications on cellular uptake," Biochimica et Biophysica Acta (BBA) - Biomembranes, vol. 1808, no. 1, pp. 332340, 2011.

[39] J. R. Komorowski, M. Tuzcu, N. Sahin et al., "Chromium picolinate modulates serotonergic properties and carbohydrate metabolism in a rat model of diabetes," Biological Trace Element Research, vol. 149, no. 1, pp. 50-56, 2012.

[40] S. Sharma, R. P. Agrawal, M. Choudhary, S. Jain, S. Goyal, and V. Agarwal, "Beneficial effect of chromium supplementation on glucose, HbA1C and lipid variables in individuals with newly onset type-2 diabetes," Journal of Trace Elements in Medicine and Biology, vol. 25, no. 3, pp. 149-153, 2011.

[41] T. Drake, K. Rudser, E. Seaquist, and A. Saeed, "Chromium infusion in hospitalized patients with severe insulin resistance: a retrospective analysis," Endocrine Practice, vol. 18, no. 3, pp. 394-398, 2012.

[42] S. R. Surani, "Severe insulin resistance treatment with intravenous chromium in septic shock patient," World Journal of Diabetes, vol. 3, no. 9, pp. 170-173, 2012.

[43] S. K. Jain, G. Kahlon, L. Morehead et al., "Effect of chromium dinicocysteinate supplementation on circulating levels of insulin, TNF- $\alpha$, oxidative stress, and insulin resistance in type 2 diabetic subjects: Randomized, double-blind, placebocontrolled study," Molecular Nutrition \& Food Research, vol. 56, no. 8, pp. 1333-1341, 2012.

[44] U. Masharani, C. Gjerde, S. McCoy et al., "Chromium supplementation in non-obese non-diabetic subjects is associated with a decline in insulin sensitivity," BMC Endocrine Disorders, vol. 12, p. 31, 2012.

[45] A. D. Wolide, B. Zawdie, T. Alemayehu Nigatu, and S. Tadesse, "Evaluation of serum ferritin and some metal elements in type 2 diabetes mellitus patients: comparative cross-sectional study," Diabetes, Metabolic Syndrome and Obesity: Targets and Therapy, vol. Volume 9, pp. 417-424, 2016.

[46] F. A. Khan, N. Al Jameil, S. Arjumand et al., "Comparative Study of Serum Copper, Iron, Magnesium, and Zinc in Type 2 Diabetes-Associated Proteinuria," Biological Trace Element Research, vol. 168, no. 2, pp. 321-329, 2015.

[47] F. Duan, Y. Li, F. Yang, and Y. Wang, "Study on iron metabolism in type 2 diabetes patients," Journal of Modern Laboratory Medcine, vol. 30, pp. 151-152, 2015.

[48] Q. Ling, R. Xu, S. Wang et al., "Correlation of serum levels of trace elements withglycometabolic parameters in patients with type 2 diabetes," Chinese Journal of Multiple Organ Diseases in the Elderly, vol. 14, pp. 440-443, 2015.

[49] M. Basaki, M. Saeb, S. Nazifi, and H. A. Shamsaei, "Zinc, copper, iron, and chromium concentrations in young patients with type 2 diabetes mellitus," Biological Trace Element Research, vol. 148, no. 2, pp. 161-164, 2012.

[50] A. Dogukan, N. Sahin, M. Tuzcu et al., "The effects of chromium histidinate on mineral status of serum and tissue in fat-fed and streptozotocin-treated type II diabetic rats," Biological Trace Element Research, vol. 131, no. 2, pp. 124-132, 2009.

[51] H. Staniek and Z. Krejpcio, “The effects of supplementary Cr3 (chromium(III) propionate complex) on the mineral status in healthy female rats," Biological Trace Element Research, vol. 180, no. 1, pp. 90-99, 2017.

[52] W. Feng, B. Li, J. Liu et al., "Study of chromium-containing proteins in subcellular fractions of rat liver by enriched stable isotopic tracer technique and gel filtration chromatography," Analytical and Bioanalytical Chemistry, vol. 375, no. 3, pp. 363368, 2003.

[53] B. J. Clodfelder and J. B. Vincent, "The time-dependent transport of chromium in adult rats from the bloodstream to the urine," JBIC Journal of Biological Inorganic Chemistry, vol. 10, no. 4, pp. 383-393, 2005.

[54] M. Ani and A. A. Moshtaghie, "The effect of chromium on parameters related to iron metabolism," Biological Trace Element Research, vol. 32, no. 1-3, pp. 57-64, 1992.

[55] B. Sundaram, K. Singhal, and R. Sandhir, "Ameliorating effect of chromium administration on hepatic glucose metabolism in streptozotocin-induced experimental diabetes," BioFactors, vol. 38, no. 1, pp. 59-68, 2012.

[56] J. B. Vincent, "Chromium: is it essential, pharmacologically relevant, or toxic?" Metal Ions in Life Sciences, vol. 13, pp. 171198, 2013. 


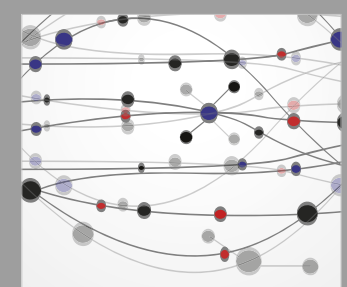

The Scientific World Journal
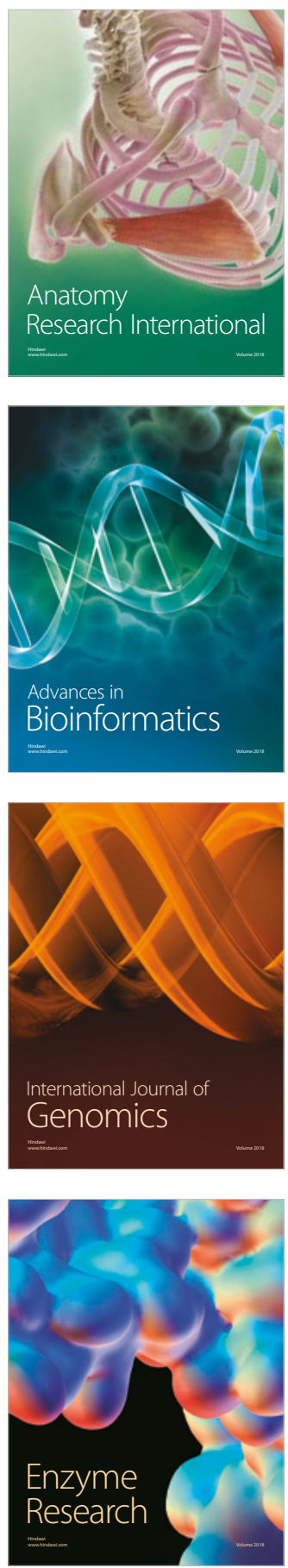
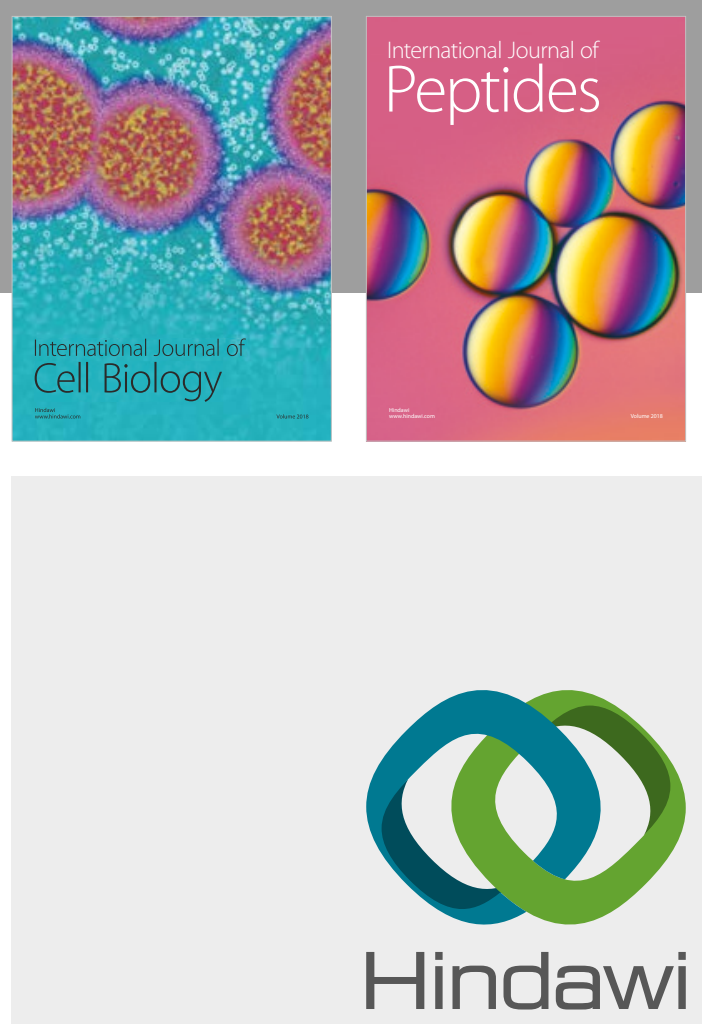

Submit your manuscripts at

www.hindawi.com
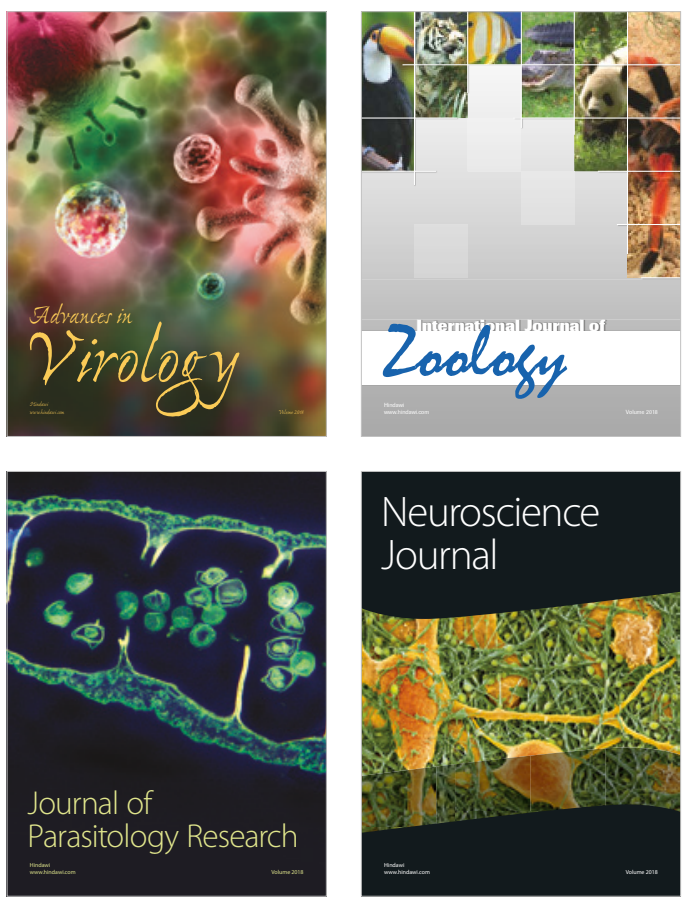
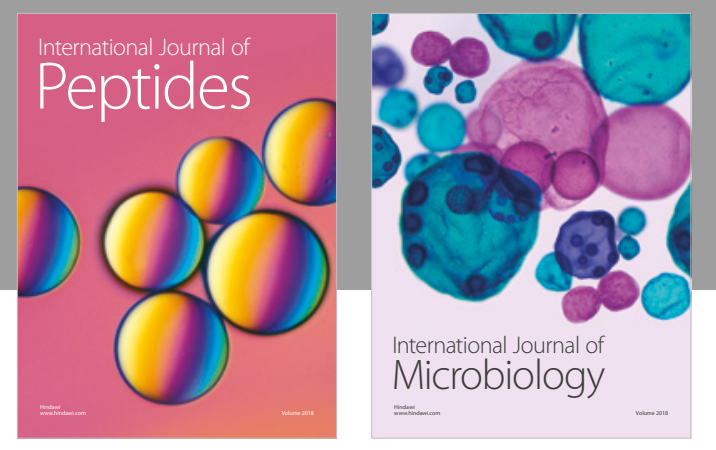

nternational Journal of Microbiology
Journal of
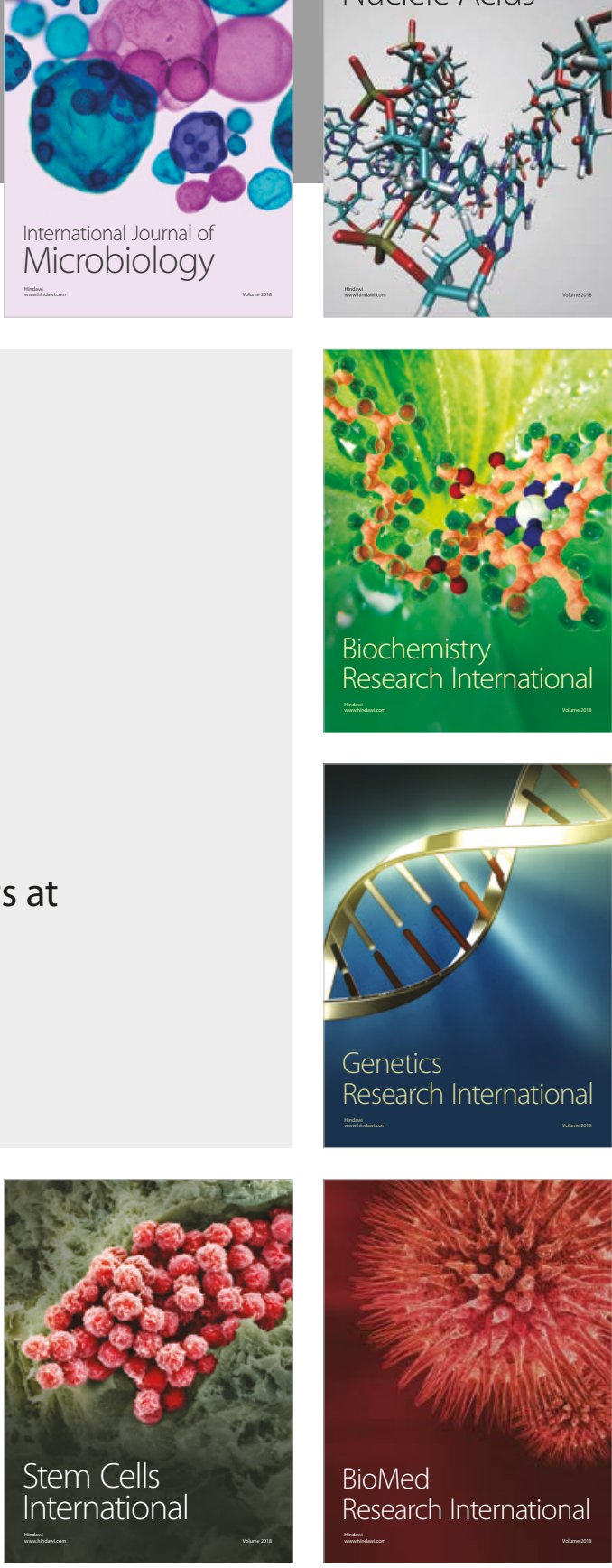
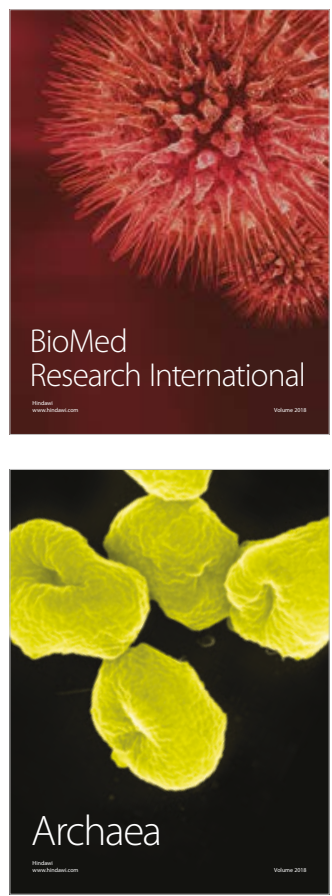